\title{
Electrochemical Impedance Spectroscopy in Interfacial Studies
}

Tamás Pajkossy ${ }^{\mathrm{a}}$ and Rafal Jurczakowski ${ }^{\mathrm{b}}$

a-Institute of Materials and Environmental Chemistry, Research Centre for Natural Sciences, Hungarian Academy of Sciences, Magyar tudósok körútja 2, Budapest, Hungary, H-1117;

e-mail: pajkossy.tamas@ttk.mta.hu

b- University of Warsaw, Faculty of Chemistry, Biological and Chemical Research Centre,

Żwirki i Wigury 101, PL-02-089 Warsaw, Poland;

email: rafjur@chem.uw.edu.pl

Keywords: interface, double layer, semiconductors, impedance, capacitance, constant phase element

\section{Abstract}

An important role of the electrochemical impedance spectroscopy (EIS) is the characterization of the electrical double layer formed at the electrode/electrolyte interfaces. The phenomenological double layer studies with an aqueous and ionic liquid electrolytes are reviewed with a conclusion that the double layer capacitance is frequency dependent as the rule rather than the exception. We discuss the impedance consequences of the nonuniform current distribution along the electrochemical interface, which also contributes to the apparent frequency dependence of the capacitance. Finally we show recent articles on nonconventional EIS techniques with high lateral resolution or enabling fast measurements.

\section{Introduction}

A principal role of the electrochemical impedance spectroscopy (EIS) - in the context of interfaces - is the characterization of the double layer structures on the electrode/electrolyte interfaces; the key concept of the double layer capacitance is in the focus of this review. Accordingly, the first section deals with related, mostly phenomenological, studies with aqueous and ionic liquid electrolytes. We summarize the experimental evidences that the double layer's differential capacitance, in general, is frequency dependent; this dependence - often named as capacitance dispersion - is an important aspect in studies of electrode processes and electrode materials. Non-uniform current distribution along the interface also leads to a capacitance dispersion, however, this effect is not related to the inherent properties of the interface.-For the possible causes of the dispersion, see Table 1 . Since various causes of the dispersion may coexist in real experimental systems in a hardly separable way, we concentrate the measurements done with systems of well-defined interfaces of single crystalline metals in contact with ionic liquids or aqueous solutions.

Finally we show recent articles related to non-conventional EIS methods since the development of such techniques can also contribute to the elucidation of the complex nature of the double layer.

Because the impedances, $Z(\omega)$, of capacitive systems are typically very large at low frequencies, the interfacial capacity can be preferably visualized in a complex plane, in which the imaginary part of the complex capacitance is plotted against the real part by analogy to representation used in studies of dielectrics [1]. However, in this representation the electrochemical impedance has to be corrected for serial (typically electrolyte) resistance $R_{s} \equiv \operatorname{Re}(Z(\omega \rightarrow \infty))$, so that by the definition equation $C(\omega) \equiv 1 /\left[j \omega\left(Z(\omega)-R_{S}\right)\right]$ where $j$ and $\omega$ are the imaginary unit and the angular frequency, respectively. Note that in the absence of faradaic reactions, charging of an ideal "electrostatic" double layer yields to $Z(\omega)$ as if $C$ were frequency-independent. Hence, the frequency dependence of $C(\omega)$ is a good measure of the deviation from the ideal capacitive behaviour. However, parameters of the equivalent circuits should be fitted to actually measured immittance (typically impedance), rather than to $C(\omega)$. 


\section{Double layer capacitance measurements}

\section{Metal/lonic liquid interfaces}

The double layer studies of the metal|ionic liquid (IL) interface started about a decade ago with the question of how the interfacial differential capacitance depends on the electrode potential, $E$, and whether or not this $C(E)$ function fits to the Kornyshev's respective theory [2]. Despite quite intensive studies described in about dozens of related papers, we still cannot give a convincing answer. Beside experimental difficulties this is because we have faced the problem associated with the frequency dependence of measured capacitances, $C(\omega)$. The related - mostly phenomenological - studies can be summarized as follows:

1. Silva and coworkers $[3,4]$ measured impedance spectra for polycrystalline Au, Pt, glassy carbon and $\mathrm{Hg}$ electrodes in three different ILs. From these spectra the $C(E)$ functions were evaluated as if the interfacial impedance were constant phase elements (CPEs), which makes these $C(E)$ functions difficult to be interpreted.

2. Gnahm et al. measured impedance for $A u(100)$ electrode in $\mathrm{BMIPF}_{6}$ [5] and other ILs. The capacitance spectra (Fig.1) were two-arcs shaped, corresponding to a three-branched equivalent circuit shown in Fig. 1 . When repeating these measurements with HOPG electrode, the lowfrequency arc disappears and therefore the $Z_{2}$ element in the equivalent circuit can be omitted. As evidenced by in-situ STM imaging, contrary to a rigid and inert structure of the HOPG surface, the $\mathrm{Au}(100)$ surface in contact with ILs undergoes a slow rearrangement phenomena and a distinct corrosion processes. These findings explain the presence and absence of of $Z_{2}$ in the equivalent circuit for $\mathrm{Au}(100)$ and HOPG, respectively [6].

3. Anderson et al. measured impedance for $\mathrm{Bi}(111)$ in $\mathrm{EMIBF}_{4}$ [7]. These spectra could be fitted to a two-branched equivalent circuit similar to that shown in the inset to Fig.2.

4. Atkin et al [8] measured impedance for $A u(111)$ electrode in two ionic liquids obtaining apparently good quality data. However, by fitting Cole-Cole functions to the frequency normalized admittances of the electrodes instead of the interfaces, the electrolyte resistance blurred the determined capacitances. The same applies also for other impedance measurements from the same group [9] and to the measurements of Li et al [10] for $\mathrm{Ag}(111)$ electrode in EMITFSI.

5. Small and Wheeler [11] raised important questions regarding the impedance measurements of metal|ILs interfaces as well as the assumptions underlying analysis of the results. They compared capacitance functions obtained for one and the same system by using four different methods of capacitance measurement and data analysis - yielding four different sets of results. This is a loud warning that proper experiments and appropriate analyses are crucial in impedance studies.

With the exception of capacitances influenced by slow corrosion/rearrangement processes of the electrodes, a frequency dependent double layer capacitance, $C(\omega)$, represents a single arc on the complex capacitance plane as illustrated in Fig.2. Such a spectrum can be modelled by the two-branched circuit shown in the inset. As the interfacial capacitance has a complicated frequency dependence, $C(\omega)$, the results of the numerous single-frequency capacitance measurements of the past decade are misleading. The same statement applies for the studies, in which the capacitance has been extracted from the impedance spectra by an oversimplified model, like in Refs [3] and [4]. It should be also mentioned that the impedance data for the $\mathrm{Au}$ electrodes measured at low frequencies $(<100 \mathrm{~Hz})$ are particularly dubious because these capacitances are additionally affected by the corrosion processes of the gold surfaces. 


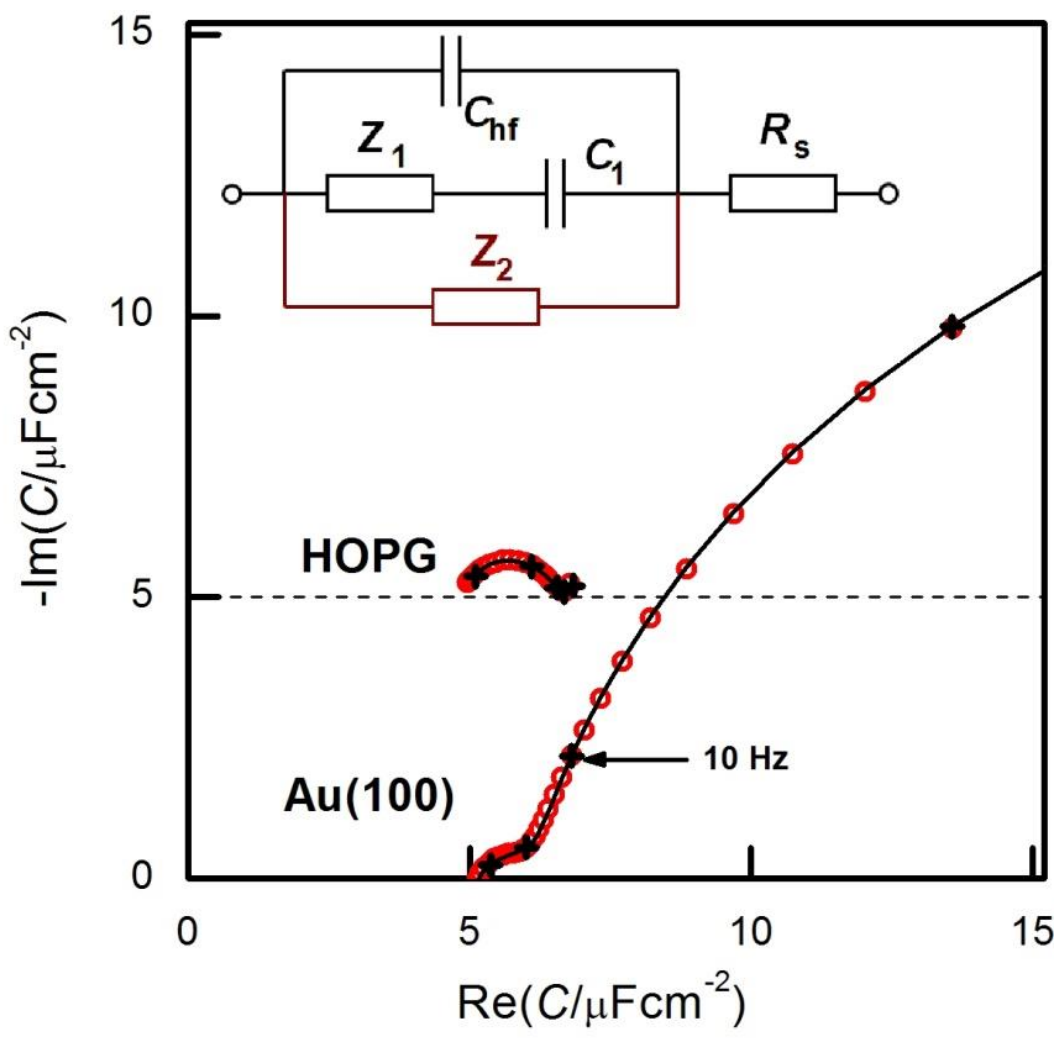

Fig.1: a. Interfacial capacitance spectra $(1 \mathrm{kHz}-1 \mathrm{~Hz})$ of $\mathrm{Au}(100)$ and $\mathrm{HOPG}$ in BMIPF $\mathrm{F}_{6}$ : measured data points (open symbols) combined with fitted ones (solid lines). The HOPG spectrum is shifted along the ordinate for clarity. The interfacial capacitance spectra, $C(\omega)$ is calculated from the measured $Z(\omega)$ impedance spectra as $\left.C(\omega) \equiv 1 /\left\{Z(\omega)-R_{s}\right)\right\}=$ $1 /\{Z(\omega)-Z(\omega \rightarrow \infty)\}$. Inset: empirical equivalent circuit, for metal/ionic liquid interfaces. $Z_{1}$ was often found to be a serial $R-W$ (Warburg element) combination; $Z_{2}$ is typically a $R-W$-C series or its absent (see text for explanation).

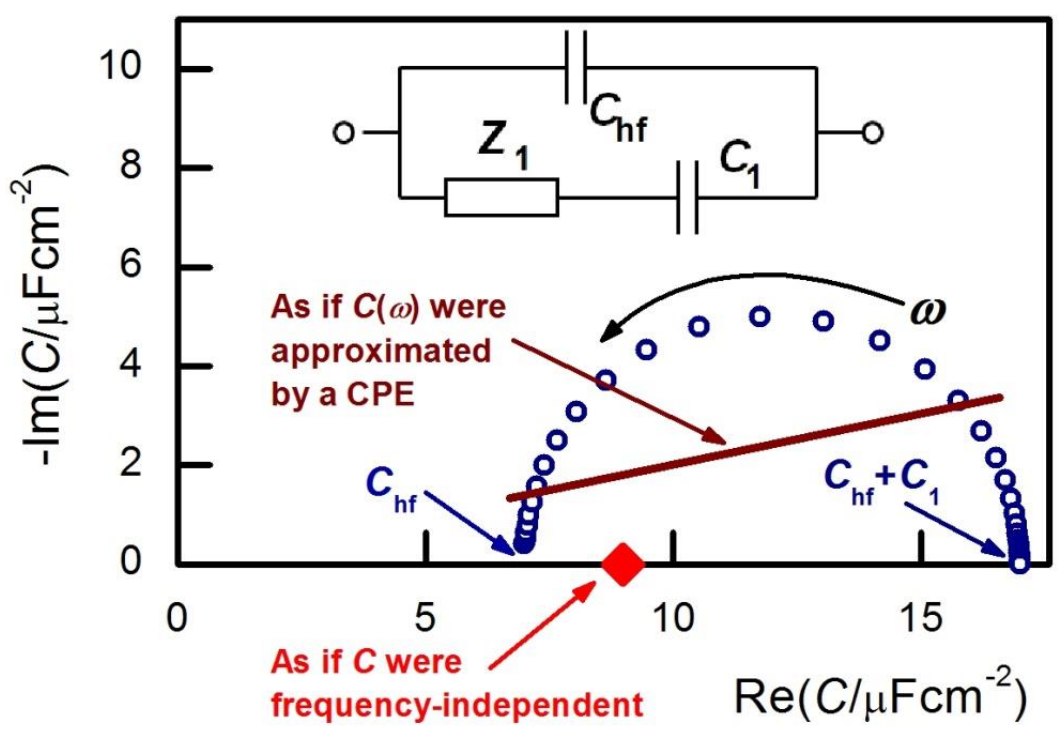

Fig.2: Illustration of capacitance spectra of metal/IL interfaces, and the corresponding empirical equivalent circuit. For $Z_{1}$ see the legend of Fig.1. For comparison, the point-like spectrum of an ideal capacitance and the straight line spectrum of a CPE are also included. 
With these considerations the double layer capacitance has been found to be similar for $\mathrm{Au}(100)$ and HOPG in the same ionic liquid. The high frequency limit of $C(\omega)$ is about 4-6 uF/ $\mathrm{cm}^{2}$ for the ionic liquids studied; for ILs with smaller ions it is larger. Neither $C_{\mathrm{hf}}$ (the high frequency limit) nor $C_{\mathrm{hf}}+C_{1}$ (the low frequency limit) appeared similar to what predicted by Kornyshev's model [2].

\section{Semiconductor/aqueous solution interfaces}

The double layer on semiconductor-electrolyte junctions is traditionally analyzed in terms of the MottSchottky theory yielding a certain $C(E)$ relation for the depletion region potentials. In principle, this theory applies for weakly doped semiconductors in contact with high concentration electrolytes in the absence of interfacial processes. In practice, this theory is often used for any semiconductor electrodes; with many and diverse corrections yielding complicated equivalent circuits introduced to eliminate the apparent frequencydependence of the Mott-Schottky lines. A rare exception is a recent characterization of the boron-doped diamond (BDD) samples [12] in neutral aqueous solution: In case of boron-doped diamond with low doping $(<=2000 \mathrm{ppm}$ ) and hydrogen-terminated surface, within the water stability range the interfacial impedance was found to be a CPE of almost-unity exponent (>0.95) and the Mott-Schottky plot exhibited a fairly broad (approx $0.4 \mathrm{~V}$ ) potential range of linearity. Acceptor concentration obtained from the slope of the MottSchottky plot was in order-of-magnitude agreement with the values obtained from neutron depth profiling and Raman spectroscopy. With higher doping levels and for oxygen terminated samples the Mott-Schottky analyses yielded less coherent results.

BDD's surface is hydrogen terminated and oxygen terminated at sufficiently negative and positive potential, respectively. The change between these states can be followed by measuring EIS while slowly scanning the potential. Such a "dynamic EIS", (DEIS, see Section 3) series, performed in sulphuric acid solution has been shown in [13]. Along with the results of various surface analyses the authors could demonstrate that the oxidation of termination bonds is a multistep process, with each step starting at different polarization potential.

\section{Metal/aqueous solutions interfaces}

Ion adsorption often causes frequency-dependent interfacial capacitances as in the classical cases of the reaction rate controlled hydrogen adsorption [14], diffusion-controlled adsorption of organic substances [15] or dense anion adlayer formation [16]. Tymoczko et al [17] showed a new application of the EIS for detecting very slow adlayer transformations on Pt(111) electrode in sulphuric acid solutions within the $0.5 \mathrm{~V}<\mathrm{E}$ vs RHE $<1 \mathrm{~V}$ potential range. Although, the cyclic voltammograms for this system is generally featureless, a small asymmetric hump appears at the potential about $0.7 \mathrm{~V}$; in this region bisulphate anions form the co-existing $(\sqrt{ } 3 \times \sqrt{ } 7)$ and $(1 \times 3)$ superstructures and the hump is attributed to a phase transition between these two superstructures [18]. At $\mathrm{E}<0.55 \mathrm{~V}$ (negative with respect to the "butterfly peak") the interface is ideally capacitive, whereas at positive potentials to the peak, the interface behaves as a CPE of with an exponent significantly smaller than unity; the CPE exponent has a minimum of $<0.9$ close to the location of the hump. The CV hump and the less-than-unity CPE exponent are the manifestation of one and the same surface process, i.e. to the slow rearrangements of the above two superstructures.

Formation and structure of organic adlayers of 4,4'-bipyridine on $\mathrm{Cd}(0001)$ in a neutral aqueous sulphate solutions has been studied by Pikma et al [19]. On $\mathrm{Cd}(0001)$ there is a potential region between that of the surface oxide formation and the hydrogen evolution where 4,4'-bipyridine is adsorbed as demonstrated by voltammetry and capacitance measurements. In this potential range the impedance spectra was interpreted in terms of the diffusion-controlled adsorption impedance theory with certain modifications [15]. In a narrow potential range almost full coverage was observed and the presence of adlayer was nicely demonstrated by in-situ STM images displaying bipyridine rows. 


\section{Origin of the frequency dependence of the interfacial capacitance}

Disregarding the rare exceptions, double layer capacitance is a frequency-dependent quantity, often approximated by a constant-phase element (CPE). This empirical element, however, gives little if any information on the physical origin of the dispersion. As summarized in Table 1, the frequency dependence can be attributed solely to the double layer or to a coupling between solution resistance and an interfacial (frequency-independent) capacitance. The $C(\omega)$ dependencies discussed in the previous section has to be ascribed to a double layer rearrangement process since it was observed on a planar and energetically uniform surface of single crystals. In this section the other cases will be analyzed when the conditions of infinite planar surface and/or energetic homogeneity do not hold, yielding to current density inhomogeneity along the electrode surface.

\begin{tabular}{|c|c|c|c|c|}
\hline \multicolumn{5}{|c|}{ Frequency dispersion of the interfacial capacitance } \\
\hline Nature & \multicolumn{2}{|c|}{ Physicochemical } & \multicolumn{2}{|c|}{ Apparent } \\
\hline Location & \multicolumn{2}{|c|}{ Interface } & \multicolumn{2}{|c|}{ Interface and bulk } \\
\hline Origin & \multicolumn{2}{|c|}{$\begin{array}{l}\text { Adsorption or rearrangement processes } \\
\text { in the interfacial layer }\end{array}$} & \multicolumn{2}{|c|}{$\begin{array}{l}\text { Coupling of electrolyte resistance } \\
\text { with interfacial impedance }\end{array}$} \\
\hline Scale & \multicolumn{2}{|c|}{ atomic } & microscopic & macroscopic \\
\hline Surface energy & homogeneous & inhomogeneous & & \\
\hline Typical case(s) & $\begin{array}{l}\text { reaction rate or diffusion } \\
\text { controlled adsorption }[13,14] ; \\
\text { rearrangement of almost } \\
\text { complete adlayers }[15,16] \\
\text { and of the double layer in } \\
\text { ionic liquids [5] as studied on } \\
\text { well-defined surfaces }\end{array}$ & $\begin{array}{c}\text { CPE-like interfacial } \\
\text { capacitance of } \\
\text { rough surfaces } \\
\text { [24-26] }\end{array}$ & $\begin{array}{c}\text { porous electrode } \\
{[19,20]}\end{array}$ & $\begin{array}{c}\text { embedded disk } \\
{[22,23]}\end{array}$ \\
\hline
\end{tabular}

Table 1: Classification of the physicochemical and apparent frequency dependence of the interfacial capacitances discussed in this work.

\section{Current density non-uniformities on the microscopic and macroscopic scales}

Frequency dependence of the capacitance might be the result of a purely physical effect caused by the cell or the electrode geometry: current density is not uniform because the "effective solution resistance" varies along the surface. This is the case for the two archetypes: for porous electrodes and for the embedded disk electrode (often used as RDE).

(i) Impedance behaviour of porous electrodes has traditionally been theorized by the finite transmission line model of de Levie [20]. This year a general theory of impedance of porous electrodes has been published, [21], in which many and diverse possible Faradaic processes within the porous system had been taken into account. This theory has been claimed to be appropriate for various practical porous electrode systems. Accordingly, the fairly complicated result should be simplified to yield special cases of porous electrodes with blocking surfaces, with faradaic reactions and with insertion reactions, respectively.

(ii) On the embedded disk, especially at its edges, current density is not uniform along the surface as was shown in Newman's analysis just fifty years ago [22] for a $d c$ case. Non-uniformity appears also with $a c$ currents, causing that the impedance spectra is not simply the sum of a solution resistance and the interfacial impedance but they are somewhat coupled; in other words: non-trivial frequency dependences of the equivalent circuit elements appear. This effect has been analyzed in two recent papers in the same issue of the J. Electroanal. Chem. dedicated to B.Tribollet's contribution to EIS. The authors of Ref. [23], give an 
analytical solution for the electric field distribution (hence also for the impedance function) for the cases when the disk is covered with an intercalation or a porous layer. In the other paper, [24], the same (or similar) problems are simulated numerically - calculating the field distribution mostly by finite element methods. Ref. [24] is, in fact, the summary of the authors many papers on these simulations done in the past decade for many and diverse types of boundaries. As both group of authors conclude, coupling of electrolyte resistance and the interfacial impedance causes a little effect on impedance; this effect should be considered in certain special cases. As a thumb's rule for capacitive disks: the larger the disk and smaller the electrolyte's conductivity, the lower the frequency range of the dispersion. Hence, for reduction of this effect use small electrodes, preferably in hanging meniscus configuration. In the same vein, this effect, for electrodes with microscopic roughness on the micron and sub-micron scale, yields to complex frequency dependence of capacitance at high frequencies only, as it was discussed decades ago by de Levie [25] and Pajkossy [26] and was again demonstrated by numeric simulations recently [27].

\section{Geometrical irregularities on atomic scale}

Frequency dependence of the capacitance is often observed for polycrystalline surfaces; in particular, if the surface is rough. The dominant explanation in the second half of the past century was a purely physical one, just like for porous electrodes: solution resistance and interfacial capacitance are coupled to each other. Twenty years ago Pajkossy hypothesized that microscopic roughness affects capacitance dispersion in an indirect way, through energetic inhomogeneities; results of a few experiments supported that hypothesis $[26,28]$. Now Cordoba-Torres et al [29] re-checked this hypothesis: They analyzed the EIS response of a wellcontrolled electrochemical reaction taking place on a surface electrode that was progressively modified by electrodissolution. In addition, a complete characterization of the surface structure was done by means of various physical methods. Their results support the view that the CPE behavior (or in general: frequency dependence of capacitance) results from the energetic inhomogeneities which in turn affect kinetics of the omnipresent ion adsorption processes.

\section{New or non-conventional experimental methods of EIS}

Various scanning methods exist for the characterization of lateral inhomogeneities of electrodes. In these techniques, the investigated electrode surface is scanned with a test microelectrode at a close proximity. During the scans some electric quantity is measured; for a recent development and nice EIS version see [30]. Lateral resolution is determined not only by the microelectrode distance but also by the activity of the neighborhood of the locally probed area. This problem has been circumvented by the arrangement of droplet cell developed by Lohrengel and coworkers about two decades ago [31]. Using this cell, the electrochemical measurements (typically current, CV or EIS) is confined to a well-defined spot in the order of magnitude of $0.1 \mathrm{~mm}$ in diameter. A microscopic imaging technique is based on the lateral scanning of this cell, called scanning droplet cell microscopy (SDCM). This technique, focused on the localized version of EIS is overviewed in a recent publication [32]. Illustration examples of the method are the determination of dielectric properties of oxides of various valve metal alloys.

The usual - commercially available - method of impedance measurement employs sinusoidal perturbation of potential or current and the resulting sinusoidal signals of current and potential are analyzed. For measuring the spectra, the frequency is varied stepwise. This method is precise though somewhat timeconsuming. An alternative method employs steady state multi-frequency perturbation in the form of sum of harmonics [33]. The potential and current signals are Fourier-transformed and impedances are calculated for each frequencies. We note that methods employing other forms of multi-frequency perturbation like white noise [34], wavelets [35], or square-waves [36] also exist. These multiple-fequency variants of EIS - are much 
faster than the conventional one (on the expense of lower precision) and thus can be used to track temporal changes at constant potential. Such a study has been reported on the temporal changes of the kinetics of the ferrocyanide/ferricyanide reaction on gold at the equilibrium potential [37] - the finding is that an ironcyanide layer is formed slowly on the gold making the charge transfer slower.

Due to the fast nature of multiple frequency EIS, it can be performed also when the potential is scanned (with certain conditions on the relation of minimum frequency and scan rate). With this method called dynamic EIS (DEIS) the advantages of CV and EIS can be coupled; for details see [38]. With such a simultaneous CV and EIS the danger of surface contaminations can be decreased. This was the case when the rearrangement of sulphate adlayer on $\mathrm{Pt}(111)$ has been studied [17]; and at the determination of the capacitance at the gold-ionic liquid interface [11].

\section{Concluding comments}

1. In general, double layer capacitance is frequency dependent, revealed as arc(s) - or as a CPE approximation: a line - on the complex plane plot of $C(\omega)$. It stems from the physicochemical nature of the processes therein, rather than from electrostatics only. Physicochemical models should be developed in order to get deeper insight in the dynamics of the double layer rearrangements.

2. Development of dynamic impedance spectroscopy and local impedance techniques can contribute to the elucidation of the nature of studies when the systems get spoiled fast or topographic information is needed. Improvements are welcome.

\section{Acknowledgements:}

The authors apologize Authors whose work has not been cited owing to the limited space of the present article. The support of Hungarian funding agency OTKA (under contract no. 112034) is highly appreciated

\section{References and recommended reading}

Papers of particular interest, published within the period of review (2015-2016), have been highlighted as:

- of special interest

$\bullet$ of outstanding interest

1. Cole KS, Cole RH: Dispersion and absorption in dielectrics II. Direct current characteristics. The Journal of Chemical Physics 1942, 10:98-105.

2. Kornyshev AA: Double-layer in ionic liquids: paradigm change? J Phys Chem B 2007, 111:5545-5557.

3. Gomes C, Costa R, Pereira CM, Silva AF: The electrical double layer at the ionic liquid/Au and Pt electrode interface. RSC Adv 2014, 4:28914-28921.

4. Costa R, Pereira CM, Silva AF: Charge storage on ionic liquid electric double layer: the role of the electrode material. Electrochim Acta 2015, 167:421-428.

5. Gnahm M, Müller C, Répánszki R, Pajkossy T, Kolb D: The interface between Au (100) and 1-butyl-3methyl-imidazolium-hexafluorophosphate. Phys Chem Chem Phys 2011, 13:11627-11633.

6. Müller $C$, Németh $K$, Vesztergom S, Pajkossy T, Jacob T: The interface between HOPG and 1-butyl-3methyl-imidazolium hexafluorophosphate. Phys Chem Chem Phys 2016, 18:916-925.

- The double layer capacitance spectrum on the complex plane is a single arc as concluded from the comparison of EIS measurements on HOPG and $A u(100)$ in an ionic liquid.

7. Anderson E, Grozovski V, Siinor L, Siimenson C, Lust E: Comparative in situ STM, cyclic voltammetry and impedance spectroscopy study of Bi (111)| 1-ethyl-3-methylimidazolium tetrafluoroborate interface. $J$ Electroanal Chem 2015, 758:201-208. 
8. Atkin R, Borisenko N, Drüschler M, Endres F, Hayes R, Huber B, Roling B: Structure and dynamics of the interfacial layer between ionic liquids and electrode materials. Journal of Molecular Liquids 2014, 192:4454.

9. Jänsch T, Wallauer J, Roling B: Influence of Electrode Roughness on Double Layer Formation in Ionic Liquids. J Phys Chem C 2015, 119:4620-4626.

10. Li M-G, Chen L, Zhong Y-X, Chen Z-B, Yan J-W, Mao B-W: The electrochemical interface of Ag (111) in 1ethyl-3-methylimidazolium bis (trifluoromethylsulfonyl) imide ionic liquid-A combined in-situ scanning probe microscopy and impedance study. Electrochim Acta 2016, 197:282-289.

11. Small LJ, Wheeler DR: Influence of Analysis Method on the Experimentally Observed Capacitance at the Gold-Ionic Liquid Interface. J Electrochem Soc 2014, 161:H260-H263.

- A warning that the different measurement and analysis methods of the metal/ionic liquid interfacial capacitance lead to different results and conclusions.

12. Živcová ZV, Petrák V, Frank O, Kavan L: Electrochemical impedance spectroscopy of polycrystalline boron doped diamond layers with hydrogen and oxygen terminated surface. Diam Relat Mater 2015, 55:70-76.

- Convincing analysis of the semiconductor/electrolyte interface and Mott-Scottky plots are presented for the oxygen- and hydrogen-terminated boron-doped diamond.

13. Ryl J, Burczyk L, Bogdanowicz R, Sobaszek M, Darowicki K: Study on surface termination of boron-doped diamond electrodes under anodic polarization in $\mathrm{H}_{2} \mathrm{SO}_{4}$ by means of dynamic impedance technique. Carbon 2016, 96:1093-1105.

14. Dolin P, Ershler B: Kinetics of processes on the platinum electrode, Acta Physicochim URSS 1940, 13:747778.

15. Frumkin A, Melik-Gaykazyan V: Determination of the kinetics of organic substances by measuring the capacitance and conductivity at the electrode-solution boundary. Dokl Akad Nauk USSR: 1951:855-858.

16. Pajkossy $T$, Kolb D: Anion-adsorption-related frequency-dependent double layer capacitance of the platinum-group metals in the double layer region. Electrochim Acta 2008, 53:7403-7409.

17. Tymoczko J, Colic V, Bandarenka AS, Schuhmann W: Detection of 2D phase transitions at the electrode/electrolyte interface using electrochemical impedance spectroscopy. Surf Sci 2015, 631:81-87.

- - On Pt(111) in sulfuric acid solution the phase transitions of the sulphate adlayer cause the lowering of the CPE exponents.

18. Braunschweig Br, Daum W: Superstructures and Order- Disorder Transition of Sulfate Adlayers on Pt (111) in Sulfuric Acid Solution. Langmuir 2009, 25:11112-11120.

19. Pikma P, Kasuk H, Oll O, Ivaništšev V, Romann T, Grozovski V, Lust K, Lust E: Adsorption of 4,4'bipyridine on the $\mathrm{Cd}(0001)$ single crystal electrode surface. Electrochim Acta 2015, 180:965-976.

20. de Levie R: On porous electrodes in electrolyte solutions-IV. Electrochim Acta 1964, 9:1231-1245.

21. Huang J, Zhang J: Theory of Impedance Response of Porous Electrodes: Simplifications, Inhomogeneities, Non-Stationarities and Applications. J Electrochem Soc 2016, 163:A1983-A2000.

- A comprehensive theory for impedance of porous electrodes - a guide how to take into account various Faradaic processes.

22. Newman J: Resistance for flow of current to a disk. J Electrochem Soc 1966, 113:501-502.

23. Tjelta $M$, Sunde $S$ : Current-distribution effects on the impedance of porous electrodes and electrodes covered with films. J Electroanal Chem 2015, 737:65-77.

- A comprehensive theory of impedance of the embedded disk - with a view to porous and insertion electrodes.

24. Wu S-L, Orazem ME, Tribollet B, Vivier V: The impedance response of rotating disk electrodes. J Electroanal Chem 2015, 737:11-22.

- A comprehensive set of simulations for the impedance of the embedded disk - a guide how to take into account various Faradaic processes.

25. de Levie R: The influence of surface roughness of solid electrodes on electrochemical measurements. Electrochimica Acta 1965, 10:113-130.

26. Pajkossy T: Impedance of rough capacitive electrodes. J Electroanal Chem 1994, 364:111-125. 
27. Alexander $\mathrm{CL}$, Tribollet $\mathrm{B}$, Orazem ME: Influence of Micrometric-Scale Electrode Heterogeneity on Electrochemical Impedance Spectroscopy. Electrochim Acta 2016, 201:374-379.

28. Kerner Z, Pajkossy T: On the origin of capacitance dispersion of rough electrodes. Electrochim Acta 2000, 46:207-211.

29. Córdoba-Torres P, Mesquita TJ, Nogueira RP: Relationship between the origin of constant-phase element behavior in electrochemical impedance spectroscopy and electrode surface structure. $J$ Phys Chem C 2015, 119:4136-4147.

- A series of experiment to demonstrate that the frequency dependence of the capacitance of the rough, ideally polarizable electrode is due to energetic inhomogenities associated with roughness rather than directly to geometry.

30. Estrada-Vargas A, Bandarenka A, Kuznetsov V, Schuhmann W: In Situ Characterization of Ultrathin Films by Scanning Electrochemical Impedance Microscopy. Anal Chem 2016, 88:3354-3362.

- Thin-layer thickness mapping - through determination of interfacial capacitances - is possible by measuring impedance spectra in a scanning electrochemical microscope.

31. Lohrengel M, Moehring A, Pilaski M: Capillary-based droplet cells: limits and new aspects. Electrochim Acta 2001, 47:137-141.

32. Hassel AW, Mardare Al: Localised electrochemical impedance spectroscopy using a scanning droplet cell microscope. J Electroanal Chem 2015, 737:93-99.

- The scanning droplet cell provides good confinement of current density lines hence impedance spectra can be analyzed just as those of macroscopic cells - but with a good lateral resolution. Impedance spectra are presented taken on valve metal alloys of varied compositions.

33. Creason SC, Smith DE: Fourier transform Faradaic admittance measurements: I. Demonstration of the applicability of random and pseudo-random noise as applied potential signals. $J$ Electroanal Chem Interf Electrochem 1972, 36:A1-A7.

34. Blanc G, Epelboin I, Gabrielli C, Keddam M: Measurement of the electrode impedance in a wide frequency range using a pseudo-random noise. Electrochim Acta 1975, 20:599-601.

35. Ragoisha GA, Bondarenko AS: Potentiodynamic electrochemical impedance spectroscopy. Electrochim Acta 2005, 50:1553-1563.

36. Pospíšil L, Fanelli N: Application of the fast Walsh-Hadamard transform and the fast Haar transform for electrochemical impedance measurements based on a periodic square-wave perturbation signal derived from Walsh-functions, J. Electroanal. Chem. 1987, 222:367-377.

37. Macía LF, Petrova M, Hubin A: ORP-EIS to study the time evolution of the [Fe(CN)6]3-/[Fe(CN)6]4reaction due to adsorption at the electrochemical interface. J Electroanal Chem 2015, 737:46-53.

38. Bandarenka AS: Exploring the interfaces between metal electrodes and aqueous electrolytes with electrochemical impedance spectroscopy. Analyst 2013, 138:5540-5554. 